\title{
Automation animal tracker using complex value neural network
}

\author{
Elly Matul Imah*, Atik Wintarti, R. Sulaiman, and Manuharawati Manuharawati \\ Universitas Negeri Surabaya, Mathematics Department, Surabaya, East Java, Indonesia
}

\begin{abstract}
Animal tracker is an important phase in animal behavior analysis. It leads to understanding how, when, and why the animal use the environmental resources, how, where, and when they interact with each other, with other species, and with their environment. Understanding the animal behavior is providing the link to population distribution which is essential for predicting the human-caused environmental change and guidance for conservation strategies. Tracking and detecting the animal is time and cost consuming. Machine Learning can relieve this burden by detecting animal automatically. Complex-Valued Neural Network is a method of Machine Learning that is challenging and interesting to be explored. This study applied of Complex-Valued Neural Network (CVNN) for animal tracking, especially in detecting the animal species. The experiment results present that CVNN is robust to recognition the animal automatically.
\end{abstract}

\section{Introduction}

The understanding of ecological systems based on very limited spatial and temporal scales is inadequate, so the ecologist needs to increase the scale of spatial dan temporal data. Solving this problem some scientist used technologies for automation spatial-temporal ecology study. Sensor networks and satellite imaging have provided valuable data for collecting biodiversity data, but analysis this data manually is hight time and cost consuming. For example, counting the population of the animal in nature is an essential tool for monitoring the health of the population and broader ecosystem [1]. Machine learning is one of development filed in computer science that is able to provide to an automation method for handling this challenge.

Animal tracker especially classification the animal is a field of ecology study that is able to be handled by machine learning. Thousands satellite photo can be annotation automatically using Machine Learning. Several researchers conducted research related to how to visualize, and analyze the ecology data automatically [2], tracking the animal using satellite imaging and detecting the animal automatically [3], analysis bird migration phenomena using machine learning [4], counting the population of zebrafish automatically using deep learning [5]. Schwager et al using GPS to analyzing the cow biological period using machine learning form the spatialtemporal data [6].

Automate species identification from satellite image data, is still challenging until nowadays. The two most important task in the process of automated animal species identification feature extraction dan classification. Feature extraction is how to take some feature that is presenting the character of data. Feature extraction commonly also knowing as dimensional reduction data [7]. Many feature extraction algorithm in machine learning, for example, principal component analysis, fast Fourier transform, wavelet transform, factor analysis, kernel PCA, and many others [8-10]. Classification tasks are how the system learns and build the models from the training datasets [11], some classification algorithms in machine learning that commonly used are Backpropagation Neural Network, Support Vector Machine, Convolution Neural Network, Adaptive Multilayers Generalized Learning Vector Quantization [12].

Real world phenomena like a digital signal, digital image preprocessing, that needs to analyze in a mathematical and geometrical relationship, cannot be express only in real number. Therefore some researcher modified the neural network by adding the complex numbers theories that known as Complex-Valued Neural Network CVNN. The basic theory of CVNN is modification algorithm of feed-forward Neural Network using complex number theory [13]. Ieroham et al modified recurrent neural network by adding complex number theory in topological learning for identification and control nonlinear system [14]. Masaki Kobayashi studies CVNN with a split activation function for handling the singularities of three layers CVNN, the result show that the proposed methods able to handling local minima problems [15]. Complex-valued also has been adding in deep neural network learning to estimate simultaneously the magnitude and phase of Short-time Fourier Transform coefficient [16].

This study using wavelet as feature extraction, and complex values neural network (CVNN) as a classification for automation animal tracker in species recognition using satellite-imaging dataset. This paper is organized as follow, section II explaining about feature extraction, Section III describes the concept of CVNN. Section IV presents the experimental setup, result, and

\footnotetext{
* Corresponding author: ellymatul@unesa.ac.id
} 
discussion the study. Section $\mathrm{V}$ presents the conclusion of the study.

\section{Method}

\subsection{Wavelet feature extraction}

The Satellite images that used in this study are RGB images, that consist of 12889 features. This is a high dimensional data, so the dimension of the data need to be reduced using feature extraction. Feature extraction that is used to reduce the dimension of the data is wavelet transform. Many researchers have reported that wavelet feature extraction has good performance for pattern recognition $[17,18]$.

In wavelet theory, selecting the appropriate mother wavelet and the number of decomposition level is an important part. The proper selection aims to retain the important part of the information on and still remain in the wavelet coefficients. The Mother wavelet that we used in this study is Daubechies. Wavelet Transform (WT) feature extraction is using time domain, therefore the image in spatial domain has to transform in the time domain. For data $f(x)$ WTis defined as:

$$
W_{s} f(x)=f(x) * \psi_{s}(x)=\frac{1}{s} \int_{-\infty}^{+\infty} f(t) \psi\left(\frac{x-t}{s}\right) d t
$$

Which s is scale factor, $\Psi_{s}(x)=\frac{1}{s} \Psi\left(\frac{x}{s}\right)$ is the dilation of a basic wavelet $\Psi(x)$ by the scaling factor $s$. Let $s=$ $2 j$ ( $j \in Z$, and $Z$ is the integer), then the WT is called dyadic WT[17]. The dyadic WT of a digital signal $f(n)$ can be calculated with Mallat algorithm as follows:

$$
\begin{aligned}
& S_{2 j} f(n)=\sum_{k \epsilon Z} h_{k} S_{2 j-1} f\left(n-2^{j-1} k\right) \\
& W_{2 j} f(n)=\sum_{k \in Z} g_{k} S_{2 j-1} f\left(n-2^{j-1} k\right)
\end{aligned}
$$

where $S_{2 j}$ is smoothing operator, $S_{2 j} f(n)=a_{j} . a_{j}$ is the low frequency coeficients that is approximatation of original signal while $W_{2 j} f(n)=d_{j}, d_{j}$ is high frequency coeficients that is the detail of original data.

the Throughout this study, data is decomposed from level 1 until level 3. After decomposition process, for example, decomposition at level 3 , namely a3 for the approximation of decomposition level $3, \mathrm{~d} 1-\mathrm{d} 3$ for details. The $\mathrm{d} 2, \mathrm{~d} 3$, represent the high-frequency coefficient of the data. Since a3 represents the approximation of the signal, it means that it contains the main feature of the data. The illustration of data decomposition using wavelet Daubechies can be seen on Fig1.

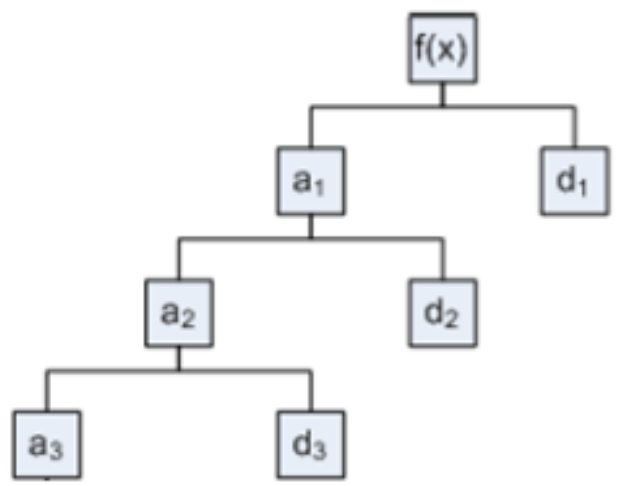

Fig. 1. Wavelet decomposition level 3.

\subsection{Complex-Valued Neural Network (CVNN) classification}

Recently there has been a rise in applications using Complex-Valued Neural Network (CVNN). This study, CVNN considered three-layers feedforward neural networks with five output neuron. The number $m$ of input neurons is fixed from feature extraction result. The architecture network of CVNN can be seen on Fig 2.

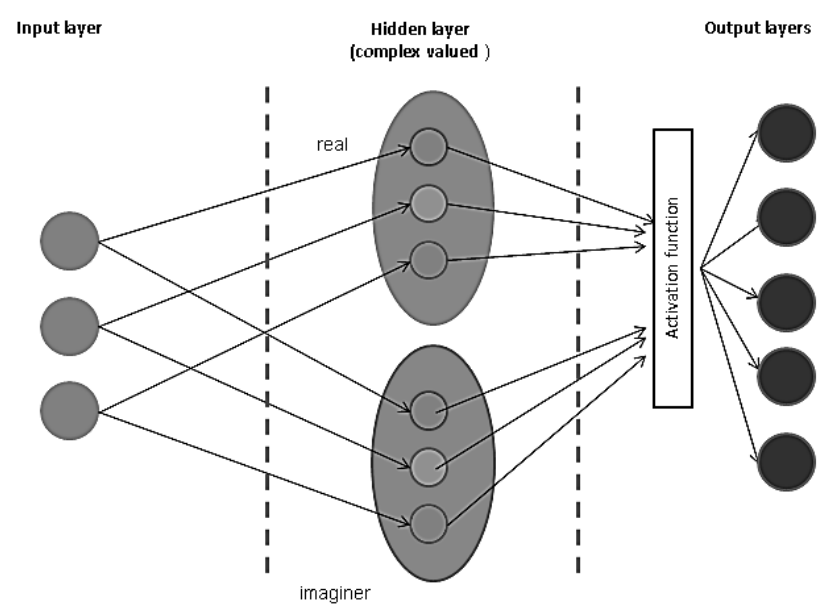

Fig. 2. The architecture network of CVNN.

Here, we briefly describe CVNN. For input vector $x=$ $\left[x_{1}+i x_{1}, x_{2}+i x_{2}, \ldots, x_{m}+i x_{m}\right]$, the weighted sum input $v_{k}(x)$ to the hidden neuron $\mathrm{k}$ are described as follow:

$$
\begin{aligned}
v_{k}(x)= & w_{k} x+\theta_{k} \\
= & \sum_{j=1}^{m}\left(w_{k j}^{R} x_{j}^{R}-w_{k j}^{i} x_{j}^{i}+\theta_{k}{ }^{R}\right) \\
& \quad+i \sum_{j=1}^{m}\left(w_{k j}^{R} x_{j}^{R}+w_{k j}^{i} x_{j}^{i}+\theta_{k}{ }^{R}\right)
\end{aligned}
$$

The output $y_{k}(x)$ of the hidden neuron $\mathrm{k}$ is described as follow:

$$
y_{k}=f_{C \rightarrow R}\left(v_{k}\right)
$$


where the activation function is defined as Eq. 4.

$$
f_{C \rightarrow R}(v)=\sqrt{\left(f_{R}\left(v^{R}\right)\right)^{2}+\left(f_{R}\left(v^{i}\right)\right)^{2}}
$$

\section{Results and discussion}

\subsection{Experimental setup}

The study uses wildebeest dataset which is downloaded from Kaggle databased center. Wildebeest dataset is an imaging satellite for monitoring the health of the population and broader ecosystem in the Serengeti National Park in Tanzania. The image in an RGB format with $64 \times 64$ pixels, five classes, wildebeest, zebra, trees, and grass. The Sample of the dataset can be seen in Fig 3.

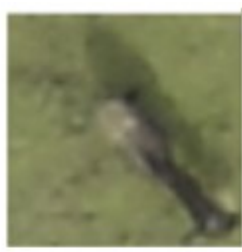

wildebeest

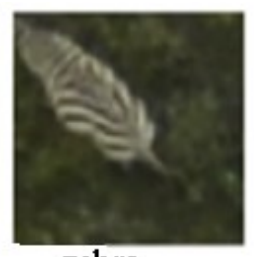

zebra

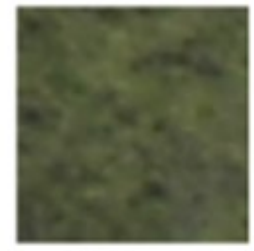

grass

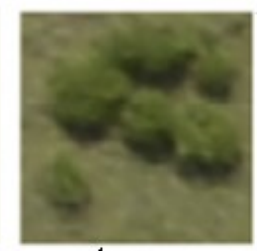

tree

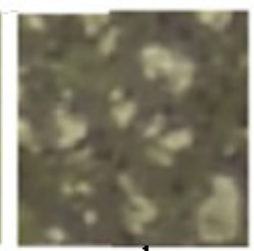

rock
Fig. 3. Sample image of wildebeest, zebra, grass, tree, and rock.

The detail of dataset distribution can be seen in Table 1 .

Table 1. Distribution of dataset.

\begin{tabular}{|c|c|c|c|c|c|}
\hline \multirow{2}{*}{ Class } & \multicolumn{2}{|c|}{ Original Dataset } & \multicolumn{2}{|c|}{ After feature extraction } \\
\cline { 2 - 5 } & & \multirow{2}{*}{ attributes } & \multicolumn{2}{|c|}{ instances } \\
\cline { 2 - 5 } & attributes & instances & & train & test \\
\hline $\mathrm{W}$ & 12288 & 50 & 1550 & 30 & 20 \\
\hline $\mathrm{Z}$ & 12288 & 50 & 1550 & 30 & 20 \\
\hline $\mathrm{g}$ & 12288 & 50 & 1550 & 30 & 20 \\
\hline $\mathrm{t}$ & 12288 & 50 & 1550 & 30 & 20 \\
\hline $\mathrm{r}$ & 12288 & 50 & 1550 & 30 & 20 \\
\hline
\end{tabular}

Table 1 shows that every class consists of fifty images, with the raw feature data is 12288 attributes, and after feature extraction, the features are reduced to 1550 attributes.

\subsection{Experimental Results}

The experiment result of the study in tracking wildebeest from satellite imaging, using wavelet feature extraction and CVNN can be seen in Table 2. As comparisson, we also run the experiment using Real-Valued Neural Netrowk (RVNN), the experiment result of RVNN can be seen in Table 3.

Table 2. Performance of wildebeest dataset classification using CVNN.

\begin{tabular}{c|llll} 
Class & Precision & Recall & F-Measure & ROC Area \\
\hline $\mathrm{w}$ & 0.833 & 0.833 & 0.833 & 0.933 \\
$\mathrm{z}$ & 0.714 & 0.882 & 0.789 & 0.958 \\
$\mathrm{~g}$ & 0.909 & 0.870 & 0.889 & 0.986 \\
$\mathrm{t}$ & 0.583 & 0.538 & 0.560 & 0.880 \\
$\mathrm{r}$ & 0.917 & 0.786 & 0.846 & 0.922 \\
\hline Weighted & 0.806 & 0.800 & 0.800 & 0.942
\end{tabular}

Table 3. Performance of wildebeest dataset classification using RVNN.

\begin{tabular}{c|llll} 
Class & Precision & Recall & F-Measure & ROC Area \\
\hline $\mathrm{w}$ & 0.824 & 0.778 & 0.800 & 0.889 \\
$\mathrm{z}$ & 0.531 & 1.000 & 0.694 & 0.890 \\
$\mathrm{~g}$ & 0.950 & 0.826 & 0.884 & 0.981 \\
$\mathrm{t}$ & 0.571 & 0.308 & 0.400 & 0.681 \\
$\mathrm{r}$ & 1.000 & 0.643 & 0.783 & 0.942 \\
\hline Weighted & 0.790 & 0.741 & 0.737 & 0.891 \\
Avg. & & & &
\end{tabular}

From Table 2 and Table 3, we can see that overall the weighted average of CVNN is better than RVNN. The ROC area of CVNN in every class almost 0.9 , difference RVNN the ROC area in every class are 0.6 to 0.9 . This result show that CVNN has better performance for tracking wildebeest than RVNN. CVNN has better performance than RVNN because CVNN able to learn the dataset more detail, therefore model that is resulted by CVNN in training phase able to generalized than RVNN.

\section{Conclusion}

This study examined the CVNN for automatically wildebeest animal tracker using satellite imaging that classifies into five classes, wildebeest, zebra, grass, tree, and rock. The experiments 1550 attributes that is resulted from wavelet feature extraction. The training and testing data ratio in this study is $3: 2$. The performance of CVNN in recognizing the five classes of wildebeest dataset better than RVNN because CVNN learns from every detail of the dataset to build the models, therefore the model able to generalization the unknown data very well.

\section{References}

1. S. Dodge et al., "The environmental-data automated track annotation (Env-DATA) system: linking animal tracks with environmental data.," Mov. Ecol., vol. 1, no. 1, p. 3, (2013)

2. A. H. Alvarado, T. L. Fuller, and T. B. Smith, "Integrative tracking methods elucidate the evolutionary dynamics of a migratory divide," Ecol. Evol., vol. 4, no. 17, pp. 3456-3469, (2014)

3. T. Burghardt, J. Ćalić, and B. T. Thomas, "Tracking 
Animals In Wildlife Videos Using Face Detection.”

4. J. Shao, Y. Zhou, J. Li, X. Wang, Z. Luo, and B. Yan, "Spatial Distribution Analysis of Wild Bird Migration in Qinghai Lake Based on Maximum Entropy Modeling," 2011 Second Int. Conf. Netw. Distrib. Comput., no. October 2011, pp. 140-144, (2011)

5. Z. XU and X. E. Cheng, "Zebrafish tracking using convolutional neural networks," Sci. Rep., vol. 7, no. January, p. 42815, (2017)

6. M. Schwager, D. M. Anderson, Z. Butler, and D. Rus, "Robust classification of animal tracking data," Comput. Electron. Agric., vol. 56, no. 1, pp. 46-59, (2007)

7. X. Wang, J. Liang, and F. Guo, "Feature extraction algorithm based on dual-scale decomposition and local binary descriptors for plant leaf recognition," Digit. Signal Process., vol. 34, pp. 101-107, (Nov. 2014)

8. G. E. Hinton and R. R. Salakhutdinov, "Reducing the dimensionality of data with neural networks.," Science, vol. 313, no. 5786, pp. 504-7, (Jul. 2006)

9. E. M. Imah, F. Al Afif, M. Ivan Fanany, W. Jatmiko, and T. Basaruddin, "A comparative study on Daubechies Wavelet Transformation, Kernel PCA and PCA as feature extractors for arrhythmia detection using SVM," in IEEE Region 10 Annual International Conference, Proceedings/TENCON, pp. 5-9. (2011)

10. J. Cheng, B. Xie, C. Lin, and L. Ji, "A comparative study in birds: call-type-independent species and individual recognition using four machine-learning methods and two acoustic features," Bioacoustics, vol. 21, no. 2, pp. 157-171, (Jun. 2012)

11. A. Iosifidis, A. Tefas, and I. Pitas, "Approximate kernel extreme learning machine for large scale data classification," Neurocomputing, (2016)

12. E. M. Imah, W. Jatmiko, and T. Basaruddin, "Electrocardiogram for biometrics by using adaptive multilayer generalized learning vector quantization (AMGLVQ): Integrating feature extraction and classification," Int. J. Smart Sens. Intell. Syst., vol. 6, no. 5, pp. 1891-1917, (2013)

13. M. Faijul Amin and K. Murase, "Single-layered complex-valued neural network for real-valued classification problems," Neurocomputing, vol. 72, no. 4-6, pp. 945-955, (2009)

14. I. S. Baruch, V. A. Quintana, and E. P. Reynaud, "Complex-valued neural network topology and learning applied for identification and control of nonlinear systems," Neurocomputing, vol. 233, no. December 2015, pp. 104-115, (2017)

15. M. Kobayashi, "Singularities of Three-Layered Complex-Valued Neural Networks With Split Activation Function," IEEE Trans. Neural Networks Learn. Syst., pp. 1-8, (2017)

16. Y. S. Lee, C. Y. Wang, S. F. Wang, J. C. Wang, and C. H. Wu, "Fully complex deep neural network for phase-incorporating monaural source separation," ICASSP, IEEE Int. Conf. Acoust. Speech Signal Process. - Proc., pp. 281-285, (2017)

17. R. Schneider and F. Kr, "Daubechies Wavelets and
Interpolating Scaling Functions and Application on PDEs," pp. 1-44, (2007)

18. J. Du, C.-M. Zhai, and Q.-P. Wang, "Recognition of plant leaf image based on fractal dimension features," Neurocomputing, vol. 116, pp. 150-156, Sep. (2013) 